\title{
Sharp lower bound for the total number of matchings of tricyclic graphs
}

\author{
Shuchao Li* \\ Faculty of Mathematics and Statistics \\ Central China Normal University \\ Wuhan 430079, P.R. China \\ lscmath@mail.ccnu.edu.cn
}

\author{
Zhongxun Zhu \\ Faculty of Mathematics and Statistics \\ South Central University for Nationalities \\ Wuhan 430074, P.R. China \\ zzxun73@163.com
}

Submitted: Mar 26, 2010; Accepted: Aug 24, 2010; Published: Oct 5, 2010

Mathematics Subject Classifications: 05C69, 05C35

\begin{abstract}
Let $\mathscr{T}_{n}$ be the class of tricyclic graphs on $n$ vertices. In this paper, a sharp lower bound for the total number of matchings of graphs in $\mathscr{T}_{n}$ is determined.
\end{abstract}

\section{Introduction}

The total number of matchings of a graph is a graphic invariant which is important in structural chemistry. In the chemistry literature this graphic invariant is called the Hosoya index of a molecular graph. It was applied to correlations with boiling points, entropies, calculated bond orders, as well as for coding of chemical structures $[12,13,26$, 32]. Therefore, the ordering of molecular graphs in terms of their Hosoya indices is of interest in chemical thermodynamics. Let $G$ be a graph with $n$ vertices and $m(G ; k)$ the number of its $k$-matchings. It is convenient to denote $m(G ; 0)=1$ and $m(G ; k)=0$ for $k>[n / 2]$. The Hosoya index of $G$, denoted by $z(G)$, is defined as the sum of all the numbers of its matchings, namely

$$
z(G)=\sum_{k=0}^{[n / 2]} m(G ; k) .
$$

The Hosoya index was introduced by Hosoya [13] and since then, many researchers have investigated this graphic invariant (e.g., see $[2,4,5,12])$. An important direction

*Financially supported by self-determined research funds of CCNU from the colleges' basic research and operation of MOE (CCNU09Y01005, CCNU09Y01018) and the National Science Foundation of China (Grant No. 11071096). 
is to determine the graphs with maximal or minimal Hosoya indices in a given class of graphs.

As for $n$-vertex trees, it has been shown that the path has the maximal Hosoya index and the star has the minimal Hosoya index (see [12]). Hou [14] characterized the trees with a given size of matching and having minimal and second minimal Hosoya index, respectively. In [22, 29], Liu and Ou, respectively, characterized the trees of diameter 4 with maximal Hosoya index. In [28], Ou characterized the trees without perfect matching having maximal Hosoya index. In [29], Ou also characterized the trees of diameter 5 with maximal Hosoya index. In [33] Yu and Lv characterized the trees with $k$ pendants having minimal Hosoya index.

As for $n$-vertex unicyclic graphs, Deng and Chen [6] gave the sharp lower bound on the Hosoya index of unicyclic graphs. In [15], Hua determined the minimum of the Hosoya index within a class of unicyclic graphs. Hua [16] also characterized the unicyclic graph with given pendants having minimal Hosoya index. In [21] Li et al. characterized unicyclic graphs with minimal, second-minimal, third-minimal, fourth-minimal, fifth-minimal and sixth-minimal Hosoya index. Recently, Ou [27] determined the unicyclic graphs with maximal Hosoya index. For $n$-vertex bicyclic graphs, Deng $[7,8]$ determined sharp upper and lower bounds on Hosoya index of bicyclic graphs, respectively.

Recently, for other class of graphs, Li et al. determined a sharp lower bound for the Hosoya index of quasi-tree graphs; see [20]. Liu and Lu characterized the cacti graphs with minimal Hosoya index in [23]. Machnicka et al. determined sharp bounds for the Hosoya index of connected graphs [25]. In [30], Ren and Zhang determined the sharp upper bound for the Hosoya index of double hexagonal chains. In [31], Shiu studied the extremal Hosoya index of hexagonal spiders.

In light of the information available for the total number of matchings of trees, unicyclic graphs, bicyclic graphs, it is natural to consider other classes of graphs, and the connected graphs with cyclomatic number 3, i.e., the set of tricyclic graphs, is a reasonable starting point for such an investigation. The tricyclic graph has been considered in mathematical and chemical literature (in total $\pi$-electron energies with the framework of the HMO approximation $[18,19]$, the theory of graphic spectra and nullity of graphs; see $[3,9,10$, $11,17]$ ), whereas to our best knowledge, the total number of matchings of tricyclic graphs was, so far, not considered. In this paper, we characterize the extremal graphs among $n$-vertex tricyclic graphs with the smallest value of total number of matchings.

In order to state our results, we introduce some notation and terminology. For other undefined notation we refer to Bollobás [1]. Recall, a connected $n$-vertex graph is tricyclic if it has $n+2$ edges. $\mathscr{T}_{n}$ denotes the set of all $n$-vertex tricyclic graphs. If $W \subset V(G)$, we denote by $G-W$ the subgraph of $G$ obtained by deleting the vertices of $W$ and the edges incident with them. Similarly, if $E \subset E(G)$, we denote by $G-E$ the subgraph of $G$ obtained by deleting the edges of $E$. If $W=\{v\}$ and $E=\{x y\}$, we write $G-v$ and $G-x y$ instead of $G-\{v\}$ and $G-\{x y\}$, respectively. Denote the neighborhood of $v \in V(G)$ by $N(v)=N_{G}(v)$; and let $N[v]=N(v) \cup\{v\}$. Throughout the paper we denote by $P_{n}, K_{1, n-1}$ and $C_{n}$ the $n$-vertex graph equals to the path, star and cycle, respectively. For two connected graphs $G_{1}, G_{2}$ with $V\left(G_{1}\right) \cap V\left(G_{2}\right)=\{v\}$, let $G=G_{1} v G_{2}$ be a graph 
defined by $V(G)=V\left(G_{1}\right) \cup V\left(G_{2}\right)$ and $E(G)=E\left(G_{1}\right) \cup E\left(G_{2}\right)$.

\section{Preliminaries}

In this section, we shall give some necessary results which will be used to obtain our main results in this paper.

Lemma $2.1([12])$. Let $G=(V, E)$ be a graph.

(i) If $u v \in E(G)$, then $z(G)=z(G-u v)+z(G-\{u, v\})$.

(ii) If $v \in V(G)$, then $z(G)=z(G-v)+\sum_{u \in N[v]} z(G-\{u, v\})$.

(iii) If $G_{1}, G_{2}, \ldots, G_{t}$ are the components of the graph $G$, then $z(G)=\prod_{j=1}^{t} z\left(G_{j}\right)$.

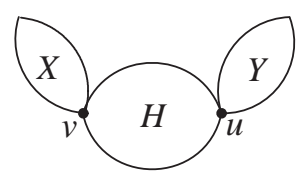

$G$

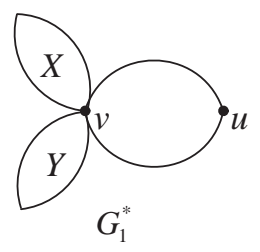

$G_{1}^{*}$

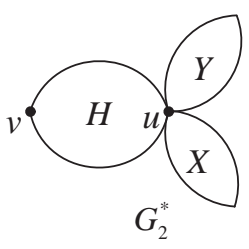

Figure 1: Graphs $G, G_{1}^{*}, G_{2}^{*}$

Two graphs are said to be disjoint if they have no vertex in common.

Lemma 2.2 ([23]). Let $H, X, Y$ be three pairwise disjoint connected graphs. Suppose that $u, v$ are two vertices of $H, v^{\prime}$ is a vertex of $X, u^{\prime}$ is a vertex of $Y$. Let $G$ be the graph obtained from $H, X, Y$ by identifying $v$ with $v^{\prime}$ and $u$ with $u^{\prime}$, respectively. Let $G_{1}^{*}$ be the graph obtained from $H, X, Y$ by identifying vertices $v, v^{\prime}, u^{\prime}$ and $G_{2}^{*}$ be the graph obtained from $H, X, Y$ by identifying vertices $u, v^{\prime}, u^{\prime}$; see Figure 1. Then

$$
z\left(G_{1}^{*}\right)<z(G) \quad \text { or } \quad z\left(G_{2}^{*}\right)<z(G) .
$$

Lemma 2.3 ([24]). Let $H$ be a connected graph and $T_{l}$ be a tree of order $l+1$ with $V(H) \cap V\left(T_{l}\right)=\{v\}$. Then $z\left(H v T_{l}\right) \geqslant z\left(H v K_{1, l}\right)$, where $v$ is the center of the star $K_{1, l}$ in $H v K_{1, l}$.

According to the definition of the Hosoya index, if $v$ is a vertex of $G$, then $z(G)>$ $z(G-v)$. In particular, when $v$ is a pendant vertex of $G$ and $u$ is the unique vertex adjacent to $v$, we have $z(G)=z(G-v)+z(G-\{u, v\})$. If set $z\left(P_{0}\right)=1$, then $z\left(P_{1}\right)=1$ and $z\left(P_{n}\right)=z\left(P_{n-1}\right)+z\left(P_{n-2}\right)$ for $n \geqslant 2$. Denote by $F_{n}$ the $n$th Fibonacci number. Recall that $F_{n}=F_{n-1}+F_{n-2}$ with initial conditions $F_{0}=1$ and $F_{1}=1$. We have

$$
z\left(P_{n}\right)=F_{n}=\frac{1}{\sqrt{5}}\left[\left(\frac{1+\sqrt{5}}{2}\right)^{n+1}-\left(\frac{1-\sqrt{5}}{2}\right)^{n+1}\right]
$$


Note that $F_{n+m}=F_{n} F_{m}+F_{n-1} F_{m-1}$, for convenience, we let $F_{n}=0$ for $n<0$.

By $[9,10,17,18,19]$, a tricyclic graph $G$ contains at least 3 cycles and at most 7 cycles, furthermore, there cannot be exactly 5 cycles in $G$. Then let $\mathscr{T}_{n}=\mathscr{T}_{n}^{3} \cup \mathscr{T}_{n}^{4} \cup \mathscr{T}_{n}^{6} \cup \mathscr{T}_{n}^{7}$, where $\mathscr{T}_{n}^{i}$ denotes the set of tricyclic graphs on $n$ vertices with exact $i$ cycles for $i=$ $3,4,6,7$. Let $G_{7}^{3}$ be a graph formed by attaching three cycles $C_{a}, C_{b}$ and $C_{c}$ to a common vertex $u$; see Figure 2. Then let $G_{n, a, b, c}^{k}$ be a graph on $n$ vertices created from $G_{7}^{3}$ by attaching $k$ pendant vertices to $u$, where $a+b+c+k=n+2$. And set

$$
\begin{gathered}
\mathscr{T}^{*}=\left\{G \in \mathscr{T}_{n}: G \text { is obtained by attaching } k\right. \text { pendant vertices } \\
\text { to one vertex except } \left.u \text {, say } v \text {, on } G_{7}^{3}\right\} .
\end{gathered}
$$

For convenience, let $\tilde{G}_{n, a, b, c}^{k}$ be any one of the members in $\mathscr{T}^{*}$. At first we shall show that the Hosoya index of any member in $\mathscr{T}^{*}$ is larger than that of $G_{n, a, b, c}^{k}$. In fact, by Lemma 2.2 , the following lemma is immediate.

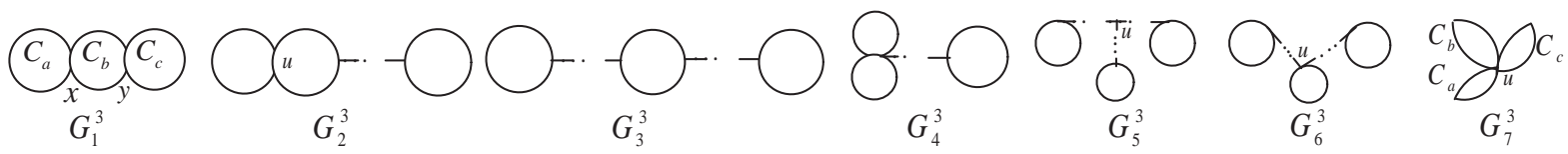

Figure 2: Seven possible cases for the arrangement of the three cycles in $\mathscr{T}_{n}^{3}$

Lemma 2.4. $z\left(\tilde{G}_{n, a, b, c}^{k}\right)>z\left(G_{n, a, b, c}^{k}\right)$.

Lemma 2.5. If $G \in \mathscr{T}_{n}^{3}$ contains exactly three cycles, $C_{a}, C_{b}$ and $C_{c}$, then $z(G) \geqslant$ $z\left(G_{n, a, b, c}^{k}\right)$.

Proof. Let $G$ be an $n$-vertex tricyclic graph processing exactly three cycles. The possible arrangements of the three cycles contained in $G$ are depicted in Figure 2; see [9, 10, 17, $18,19]$. Here we only show that our result is true when $G$ is obtained by attaching some trees to $G_{1}^{3}$; see Figure 2. With a similar method we can show that our result is also true for the other cases, i.e., $G$ is obtained by attaching some trees to $G_{i}^{3}, i=2,3,4,5,6,7$; see Figure 2. We omit the procedure here.

Let $V_{P}(G)=\left\{v \in V\left(G_{1}^{3}\right): N_{G}(v) \backslash N_{G_{1}^{3}}(v) \neq \emptyset\right\}$. If $\left|V_{P}(G)\right| \geqslant 2$, then by Lemma 2.2, we can obtain graph $G^{\prime}$ such that $G^{\prime}$ contains $G_{1}^{3}$ as its subgraph, $\left|V_{P}\left(G^{\prime}\right)\right|=\left|V_{P}(G)\right|-1$ and $z\left(G^{\prime}\right)<z(G)$. Using Lemma 2.2 repeatedly, we finally get a graph $G^{\prime \prime}$ which contains $G_{1}^{3}$ as its subgraph, $\left|V_{P}\left(G^{\prime \prime}\right)\right|=1$ and $z\left(G^{\prime \prime}\right)<z(G)$. Once again by Lemma 2.2 , we may obtain a graph $G^{*}$ such that $G^{*}$ contains $G_{7}^{3}$ (see Figure 2) as its subgraph, $\left|V_{P}\left(G^{*}\right)\right|=1$ and $z\left(G^{*}\right)<z\left(G^{\prime \prime}\right)$. By Lemma 2.3, we have either $z\left(G^{*}\right) \geqslant z\left(G_{n, a, b, c}^{k}\right)$ or, $z\left(G^{*}\right) \geqslant$ $z\left(\tilde{G}_{n, a, b, c}^{k}\right)$. Hence, in view of Lemma 2.4 , we have $z(G) \geqslant z\left(G_{n, a, b, c}^{k}\right)$, as desired. This completes the proof.

Lemma 2.6. For any positive integers $a, b, c, k$,

(i) $z\left(G_{n, a, b, c}^{k}\right)>z\left(G_{n, a-1, b, c}^{k+1}\right)$ if $a \geqslant 4, b, c \geqslant 3$.

(ii) $z\left(G_{n, a, b, c}^{k}\right)>z\left(G_{n, a, b-1, c}^{k+1}\right)$ if $b \geqslant 4, a, c \geqslant 3$. 
(iii) $z\left(G_{n, a, b, c}^{k}\right)>z\left(G_{n, a, b, c-1}^{k+1}\right)$ if $c \geqslant 4, a, b \geqslant 3$.

Proof. By symmetry, it suffices to prove (i). We omit the proofs for (ii) and (iii). By Lemma 2.1,

$$
\begin{aligned}
& z\left(G_{n, a, b, c}^{k}\right) \\
= & z\left(G_{n, a, b, c}^{k}-v\right)+\sum_{v \in N[u]} z\left(G_{n, a, b, c}^{k}-\{u, v\}\right) \\
= & z\left(P_{a-1} \cup P_{b-1} \cup P_{c-1} \cup k P_{1}\right)+2 z\left(P_{a-2} \cup P_{b-1} \cup P_{c-1} \cup k P_{1}\right)+2 z\left(P_{a-1} \cup P_{b-2}\right. \\
& \left.\cup P_{c-1} \cup k P_{1}\right)+2 z\left(P_{a-1} \cup P_{b-1} \cup P_{c-2} \cup k P_{1}\right)+k z\left(P_{a-1} \cup P_{b-1} \cup P_{c-1} \cup(k-1) P_{1}\right) \\
= & (k+1) F_{a-1} F_{b-1} F_{c-1}+2 F_{a-2} F_{b-1} F_{c-1}+2 F_{a-1} F_{b-2} F_{c-1}+2 F_{a-1} F_{b-1} F_{c-2} .
\end{aligned}
$$

Similarly,

$z\left(G_{n, a-1, b, c}^{k+1}\right)=(k+2) F_{a-2} F_{b-1} F_{c-1}+2 F_{a-3} F_{b-1} F_{c-1}+2 F_{a-2} F_{b-2} F_{c-1}+2 F_{a-2} F_{b-1} F_{c-2}$.

Thus,

$$
\begin{aligned}
z\left(G_{n, a, b, c}^{k}\right)-z\left(G_{n, a-1, b, c}^{k+1}\right)= & (k+1) F_{a-1} F_{b-1} F_{c-1}+2 F_{a-2} F_{b-1} F_{c-1}+2 F_{a-1} F_{b-2} F_{c-1} \\
& +2 F_{a-1} F_{b-1} F_{c-2}-\left[(k+2) F_{a-2} F_{b-1} F_{c-1}+2 F_{a-3} F_{b-1} F_{c-1}\right. \\
& \left.+2 F_{a-2} F_{b-2} F_{c-1}+2 F_{a-2} F_{b-1} F_{c-2}\right] \\
= & (k+1) F_{a-3} F_{b-1} F_{c-1}+2 F_{a-4} F_{b-1} F_{c-1}+2 F_{a-3} F_{b-2} F_{c-1} \\
& +2 F_{a-3} F_{b-1} F_{c-2}-F_{a-2} F_{b-1} F_{c-1} \\
\geqslant & F_{a-4} F_{b-1} F_{c-1} .
\end{aligned}
$$

Note that $a \geqslant 4, b, c \geqslant 3$, therefore by $(2.1)$,

$$
F_{a-4} F_{b-1} F_{c-1}>0
$$

and so, (i) holds. This completes the proof.

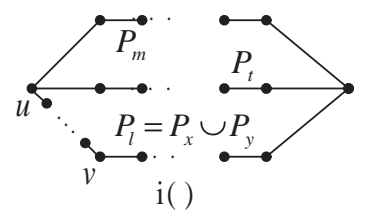

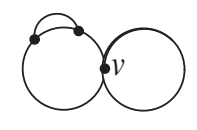

i ( i)

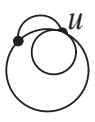

i ( ii)

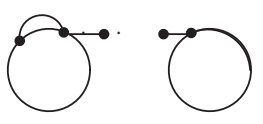

i $0 \mathrm{v}$

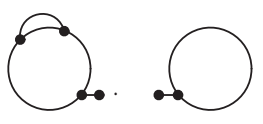

) $\mathrm{v}($

Figure 3: Four possible cases for the arrangement of the four cycles in $\mathscr{T}_{n}^{4}$

Let $P_{l}, P_{m}, P_{t}$ be three vertex-disjoint paths, where $l, m, t \geqslant 2$ and at most one of them is 2 . Identifying the three initial vertices and terminal vertices of them, respectively, the resulting graph, denoted by $B_{1}$, is called a $\theta$-graph; see Figure $3(\mathrm{i})$. Furthermore, let $C_{b}$ be a cycle. Connect $C_{b}$ and $B_{1}$ by a path $P_{s}$, where $s \geqslant 1$ and call the resulting graph $\tilde{G}$-graph. By $[9,10,17,18,19]$, we know that there are exactly four types of $\tilde{G}$-graph; see 
Figure 3(ii)-(v). Furthermore, $\mathscr{T}_{n}^{4}$ denotes the set of all graphs obtained from $\tilde{G}$-graph by attaching some trees (or nothing). For convenience, let $C_{a}, C_{c}$ and $C_{d}$ be the three cycles contained in $B_{1}$, where $C_{a}=P_{l} \cup P_{m}, C_{c}=P_{m} \cup P_{t}, C_{d}=P_{t} \cup P_{l}=P_{t} \cup P_{x} \cup P_{y}$; see Figure 3(i). Set

$$
G_{1}:=B_{1} u C_{b}, G_{2}:=B_{1} v C_{b} .
$$

Thus, we define two tricyclic graphs in $\mathscr{T}_{n}^{4}$ as follows:

- $A_{m, l, b, t}^{k}$ is an $n$-vertex tricyclic graph created from $G_{1}$ by attaching $k$ pendant vertices to $u$.

- $\bar{A}_{m, b, t}^{k, x, y}$ is an $n$-vertex tricyclic graph created from $G_{2}$ by attaching $k$ pendant vertices to $v$.

In the above two graphs, the number of pendant vertices is in fact $n+5-m-l-t-b$, i.e., $k=n+5-m-l-t-b$.

Lemma 2.7. Let $G$ be an element of $\mathscr{T}_{n}^{4}$ such that $G$ contains the $\theta$-graph $B_{1}$ and a cycle $C_{b}$ with $E\left(B_{1}\right) \cap E\left(C_{b}\right)=\emptyset$, then $z(G) \geqslant z\left(A_{m, l, b, t}^{k}\right)$, the equality holds if and only if $G \cong A_{m, l, b, t}^{k}$, where $k=n-\left(\left|V\left(B_{1}\right)\right|+\left|V\left(C_{b}\right)\right|-1\right)$.

Proof. We distinguish the following two possible cases to prove this lemma.

Case 1. $k=0$. In this case, it is sufficient for us to consider the two graphs $G_{1}, G_{2}$ defined in (2.2). Using Lemma 2.1 repeatedly, we obtain

$$
z\left(G_{1}\right)=F_{b-1} z\left(B_{1}\right)+2 F_{b-2} z\left(B_{1}-u\right), \quad z\left(G_{2}\right)=F_{b-1} z\left(B_{1}\right)+2 F_{b-2} z\left(B_{1}-v\right) .
$$

This gives

$$
z\left(G_{2}\right)-z\left(G_{1}\right)=2 F_{b-2}\left(z\left(B_{1}-v\right)-z\left(B_{1}-u\right)\right) .
$$

Furthermore,

$$
\begin{aligned}
& z\left(B_{1}-u\right)=F_{m-2} F_{l-2} F_{t-1}+F_{m-3} F_{l-2} F_{t-2}+F_{m-2} F_{l-3} F_{t-2}, \\
& z\left(B_{1}-v\right)=F_{m-2} F_{l+t-3}+F_{m-3} F_{y-2} F_{x+t-3}+F_{m-3} F_{x-2} F_{y+t-3}+F_{m-4} F_{x-2} F_{y-2} F_{t-2} .
\end{aligned}
$$

Note that $l=x+y-1$, hence

$$
\begin{aligned}
& z\left(B_{1}-v\right)-z\left(B_{1}-u\right) \\
= & F_{m-2} F_{l+t-3}+F_{m-3} F_{y-2} F_{x+t-3}+F_{m-3} F_{x-2} F_{y+t-3}+F_{m-4} F_{x-2} F_{y-2} F_{t-2} \\
& -\left(F_{m-2} F_{l-2} F_{t-1}+F_{m-3} F_{l-2} F_{t-2}+F_{m-2} F_{l-3} F_{t-2}\right) \\
= & {\left[F_{m-2} F_{l+t-3}-\left(F_{m-2} F_{l-2} F_{t-1}+F_{m-2} F_{l-3} F_{t-2}\right)\right]+F_{m-4} F_{x-2} F_{y-2} F_{t-2} } \\
& +\left(F_{m-3} F_{y-2} F_{x+t-3}+F_{m-3} F_{x-2} F_{y+t-3}-F_{m-3} F_{l-2} F_{t-2}\right) \\
= & \left(F_{m-3} F_{y-2} F_{x+t-3}+F_{m-3} F_{x-2} F_{y+t-3}-F_{m-3} F_{x+y-3} F_{t-2}\right)
\end{aligned}
$$




$$
\begin{aligned}
& +F_{m-4} F_{x-2} F_{y-2} F_{t-2} \\
= & \left(F_{m-3} F_{y-2} F_{x-1} F_{t-2}+F_{m-3} F_{y-2} F_{x-2} F_{t-3}+F_{m-3} F_{x-2} F_{y-3} F_{t}\right. \\
& \left.+F_{m-3} F_{x-2} F_{y-4} F_{t-1}-F_{m-3} F_{y-2} F_{x-1} F_{t-2}-F_{m-3} F_{y-3} F_{x-2} F_{t-2}\right) \\
& +F_{m-4} F_{x-2} F_{y-2} F_{t-2} \\
\geqslant & F_{m-3} F_{y-2} F_{x-2} F_{t-3} .
\end{aligned}
$$

By $(2.3),(2.4)$, we obtain $z\left(G_{2}\right)>z\left(G_{1}\right)$. Hence when $k=0$, we have $z(G) \geqslant z\left(G_{1}\right)=$ $z\left(A_{m, l, b, t}^{0}\right)$, the equality holds if and only if $G \cong A_{m, l, b, t}^{0}$.

Case $2 . k \geqslant 1$. In this case, by applying Lemmas 2.2 and 2.3 repeatedly, we have $z(G) \geqslant z\left(A_{m, l, b, t}^{k}\right)$, or $z(G) \geqslant z\left(\bar{A}_{m, b, t}^{k, x, y}\right)$. On the other hand, by Lemma 2.1 we have

$$
z\left(\bar{A}_{m, b, t}^{k, x, y}\right)=z\left(G_{2}\right)+k F_{b-1} z\left(B_{1}-v\right), \quad z\left(A_{m, l, b, t}^{k}\right)=z\left(G_{1}\right)+k F_{b-1} z\left(B_{1}-u\right) .
$$

This gives

$$
z\left(\bar{A}_{m, b, t}^{k, x, y}\right)-z\left(A_{m, l, b, t}^{k}\right)=\left(z\left(G_{2}\right)-z\left(G_{1}\right)\right)+k F_{b-1}\left(z\left(B_{1}-v\right)-z\left(B_{1}-u\right)\right) .
$$

By (2.6) and $z\left(G_{2}\right)>z\left(G_{1}\right)$, we have $z\left(\bar{A}_{m, b, t}^{k, x, y}\right)>z\left(A_{m, l, b, t}^{k}\right)$.

Lemma 2.8. For positive integers $m, l, x, y, b, t, k$,

(i) $z\left(A_{m, l-1, b, t}^{k+1}\right)<z\left(A_{m, l, b, t}^{k}\right)$ for either $l \geqslant 4, b \geqslant 3, m, t \geqslant 2$ and $m t \geqslant 6$, or $l=$ $3, b, m, t \geqslant 3$.

(ii) $z\left(A_{m-1, l, b, t}^{k+1}\right)<z\left(A_{m, l, b, t}^{k}\right)$ for either $m \geqslant 4, b \geqslant 3, l, t \geqslant 2$ and $l t \geqslant 6$, or $m=3, b, l, t \geqslant$ 3.

(iii) $z\left(A_{m, l, b-1, t}^{k+1}\right)<z\left(A_{m, l, b, t}^{k}\right)$ for $b \geqslant 4, l, m, t \geqslant 2$ and $\operatorname{lm} t \geqslant 18$.

(iv) $z\left(A_{m, l, b, t-1}^{k+1}\right)<z\left(A_{m, l, b, t}^{k}\right)$ for either $t \geqslant 4, b \geqslant 3, l, t \geqslant 2$ and $l t \geqslant 6$, or $t=3, m, l, b \geqslant$ 3 .

Proof. (i) Consider $B_{1}$ and $B_{1}-u$ (see Figure $3(\mathrm{i})$ ), we have

$$
\begin{aligned}
z\left(B_{1}\right)= & F_{m-2} F_{l+t-3}+F_{m-3} F_{l-2} F_{t-2}+F_{m-3} F_{l+t-3}+F_{m-4} F_{l-2} F_{t-2} \\
& +F_{m-2} F_{l+t-4}+F_{m-3} F_{l-3} F_{t-2}+F_{m-2} F_{l+t-4}+F_{m-3} F_{l-2} F_{t-3}, \\
z\left(B_{1}-u\right)= & F_{m-2} F_{l+t-3}+F_{m-3} F_{l-2} F_{t-2} .
\end{aligned}
$$

Hence, by Lemma 2.1 we get

$$
\begin{aligned}
& z\left(A_{m, l, b, t}^{k}\right) \\
= & F_{b-1} z\left(B_{1}\right)+2 F_{b-2} z\left(B_{1}-u\right)+k F_{b-1} z\left(B_{1}-u\right) \\
= & F_{b-1}\left(F_{m-2} F_{l+t-3}+F_{m-3} F_{l-2} F_{t-2}+F_{m-3} F_{l+t-3}+F_{m-4} F_{l-2} F_{t-2}+F_{m-2} F_{l+t-4}\right. \\
& \left.+F_{m-3} F_{l-3} F_{t-2}+F_{m-2} F_{l+t-4}+F_{m-3} F_{l-2} F_{t-3}\right)+2 F_{b-2}\left(F_{m-2} F_{l+t-3}\right. \\
& \left.+F_{m-3} F_{l-2} F_{t-2}\right)+k F_{b-1}\left(F_{m-2} F_{l+t-3}+F_{m-3} F_{l-2} F_{t-2}\right) .
\end{aligned}
$$


This gives

$$
\begin{aligned}
& z\left(A_{m, l, b, t}^{k}\right)-z\left(A_{m, l-1, b, t}^{k+1}\right) \\
= & F_{b-1}\left(F_{m-2} F_{l+t-5}+F_{m-3} F_{l-4} F_{t-2}+F_{m-3} F_{l+t-5}+F_{m-4} F_{l-4} F_{t-2}\right. \\
& \left.+F_{m-2} F_{l+t-6}+F_{m-3} F_{l-5} F_{t-2}+F_{m-2} F_{l+t-6}+F_{m-3} F_{l-4} F_{t-3}\right) \\
& +\left(2 F_{b-2}+k F_{b-1}\right)\left(F_{m-2} F_{l+t-5}+F_{m-3} F_{l-4} F_{t-2}\right) \\
\geqslant & F_{b-1} F_{m-3} F_{l-3} F_{t-2} .
\end{aligned}
$$

Note that at most one of $m, t$ is 2 , hence without loss of generality, let $t \geqslant 2, m \geqslant 3$; together with $b, l \geqslant 3$ yields $F_{b-1} F_{m-3} F_{l-3} F_{t-2}>0$, i.e., $z\left(A_{m, l, b, t}^{k}\right)>z\left(A_{m, l-1, b, t}^{k+1}\right)$. This completes the proof of (i).

By a similar discussion as in the proof of (i), we may also show that (ii)-(iv) are true. We omit the procedure here. This completes the proof of Lemma 2.9.

We know from $[9,10,17,18,19]$ that if a tricyclic graph has exactly six cycles, then the arrangement of these cycles has three forms; see Figure 4. Then define four tricyclic graphs in $\mathscr{T}_{n}^{6}$ as follows:

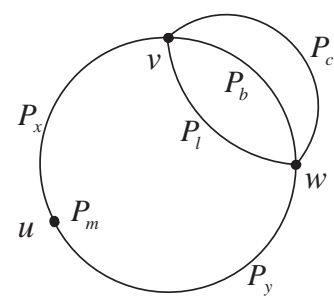

(I)

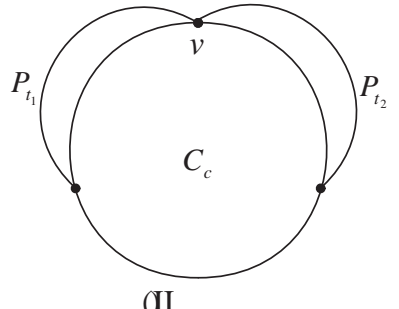

\II

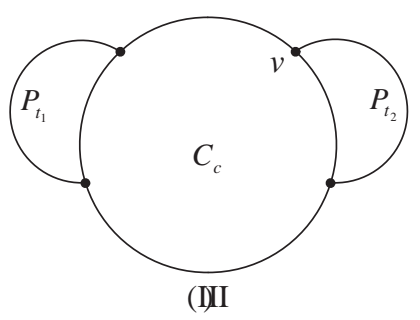

Figure 4: Three possible cases for the arrangement of the six cycles in $\mathscr{T}_{n}^{6}$

- $H_{m, l, b, c}^{k}$ is a tricyclic graph with exactly six cycles on $n$ vertices created from Figure 4(I) by attaching $k$ pendant vertices to $v(\neq u)$ of (I), where $m+l+b+c+k=n+6$ and $P_{m}=P_{x} \cup P_{y}$.

- $\bar{H}_{m, b, c}^{k, x, y}$ is any member of the set of $n$-vertex tricyclic graphs with exactly six cycles created from Figure 4(I) by attaching $k$ pendant vertices to $u(\neq v, w)$, where $m+$ $l+b+c+k=n+6$ and $P_{m}=P_{x} \cup P_{y}$.

- $Q_{c, t_{1}, t_{2}}^{k}$ is a tricyclic graph with exactly six cycles on $n$ vertices created from Figure 4(II) by attaching $k$ pendant vertices to $v$, where $c+t_{1}+t_{2}+k=n+3$.

- $S_{c, t_{1}, t_{2}}^{k}$ is a tricyclic graph with exactly six cycles on $n$ vertices created from Figure 4 (III) by attaching $k$ pendant vertices to $v$, where $c+t_{1}+t_{2}+k=n+4$.

Lemma 2.9. Let $G \in \mathscr{T}_{n}^{6}$.

(a) If the six cycles in $G$ are the same as Figure $4(I)$, then we have $z(G) \geqslant z\left(H_{m, l, b, c}^{k}\right)$. 
(b) If the six cycles in $G$ are the same as Figure $4(I I)$, then we have $z(G)>z\left(Q_{c, t_{1}, t_{2}}^{k}\right)$.

(c) If the six cycles in $G$ are the same as Figure $4(I I I)$, then we have $z(G)>z\left(S_{c, t_{1}, t_{2}}^{k}\right)$.

Proof. (a) For any graph $G \in \mathscr{T}_{n}^{6}$ that satisfies the assumption of (a), repeated applications of Lemmas 2.2 and 2.3 give

$$
z(G) \geqslant z\left(H_{m, l, b, c}^{k}\right) \text { or } z(G) \geqslant z\left(\bar{H}_{m, b, c}^{k, x, y}\right)
$$

In order to complete the proof of Lemma 2.9(a), it suffices to show that $z\left(H_{m, l, b, c}^{k}\right)<$ $z\left(\bar{H}_{m, b, c}^{k, x, y}\right)$ holds. In fact, let $v_{1}, v_{2}, \ldots, v_{k}$ be the $k$ pendant vertices of $H_{m, l, b, c}^{k}$. Set $G_{0}=$ $H_{m, l, b, c}^{k}-\left\{v_{1}, \ldots, v_{k}\right\}$. Thus,

$$
z\left(H_{m, l, b, c}^{k}\right)=z\left(G_{0}\right)+k z\left(G_{0}-v\right), \quad z\left(\bar{H}_{m, b, c}^{k, x, y}\right)=z\left(G_{0}\right)+k z\left(G_{0}-u\right) .
$$

Therefore, we have

$$
z\left(\bar{H}_{m, b, c}^{k, x, y}\right)-z\left(H_{m, l, b, c}^{k}\right)=k\left(z\left(G_{0}-u\right)-z\left(G_{0}-v\right)\right) .
$$

On the other hand,

$$
\begin{aligned}
& z\left(G_{0}-u\right) \\
= & F_{l-2} F_{b-2} F_{m+c-3}+F_{l-2} F_{b-3} F_{x-2} F_{y+c-3}+F_{l-2} F_{b-3} F_{y-2} F_{x+c-3}+F_{l-2} F_{b-4} F_{x-2} \\
& \times F_{y-2} F_{c-2}+F_{l-3} F_{b-2} F_{x-2} F_{y+c-3}+F_{l-3} F_{b-3} F_{x-2} F_{y-2} F_{c-2} \\
& +F_{l-3} F_{b-2} F_{y-2} F_{x+c-3}+F_{l-3} F_{b-3} F_{x-2} F_{y-2} F_{c-2}+F_{l-4} F_{b-2} F_{x-2} F_{y-2} F_{c-2},
\end{aligned}
$$

and

$$
z\left(G_{0}-v\right)=F_{m-2} F_{l-2} F_{b+c-3}+F_{m-2} F_{l-3} F_{b-2} F_{c-2}+F_{m-3} F_{l-2} F_{b-2} F_{c-2} .
$$

Note that $m=x+y-1$, set $\Delta:=z\left(G_{0}-u\right)-z\left(G_{0}-v\right)$, we have

$$
\begin{aligned}
\Delta= & F_{l-2} F_{b-2} F_{m+c-3}+F_{l-2} F_{b-3} F_{x-2} F_{y+c-3}+F_{l-2} F_{b-3} F_{y-2} F_{x+c-3} \\
& +F_{l-2} F_{b-4} F_{x-2} F_{y-2} F_{c-2}+F_{l-3} F_{b-2} F_{x-2} F_{y+c-3}+F_{l-3} F_{b-3} F_{x-2} F_{y-2} F_{c-2} \\
& +F_{l-3} F_{b-2} F_{y-2} F_{x+c-3}+F_{l-3} F_{b-3} F_{x-2} F_{y-2} F_{c-2}+F_{l-4} F_{b-2} F_{x-2} F_{y-2} F_{c-2} \\
& -\left(F_{m-2} F_{l-2} F_{b+c-3}+F_{m-2} F_{l-3} F_{b-2} F_{c-2}+F_{m-3} F_{l-2} F_{b-2} F_{c-2}\right) \\
= & F_{l-2} F_{b-2} F_{m-2} F_{c-1}+F_{l-2} F_{b-2} F_{m-3} F_{c-2}+F_{l-2} F_{b-3} F_{x-2} F_{y-2} F_{c-1} \\
& +F_{l-2} F_{b-3} F_{x-2} F_{y-3} F_{c-2}+F_{l-2} F_{b-3} F_{y-2} F_{x-2} F_{c-1}+F_{l-2} F_{b-3} F_{y-2} F_{x-3} F_{c-2} \\
& +F_{l-2} F_{b-4} F_{x-2} F_{y-2} F_{c-2}+F_{l-3} F_{b-2} F_{x-2} F_{y-2} F_{c-1}+F_{l-3} F_{b-2} F_{x-2} F_{y-3} F_{c-2} \\
& +F_{l-3} F_{b-3} F_{x-2} F_{y-2} F_{c-2}+F_{l-3} F_{b-2} F_{y-2} F_{x-2} F_{c-1}+F_{l-3} F_{b-2} F_{y-2} F_{x-3} F_{c-2} \\
& +F_{l-3} F_{b-3} F_{x-2} F_{y-2} F_{c-2}+F_{l-4} F_{b-2} F_{x-2} F_{y-2} F_{c-2}-\left(F_{m-2} F_{l-2} F_{b-2} F_{c-1}\right. \\
& \left.+F_{m-2} F_{l-2} F_{b-3} F_{c-2}+F_{m-2} F_{l-3} F_{b-2} F_{c-2}+F_{m-3} F_{l-2} F_{b-2} F_{c-2}\right) \\
= & F_{l-2} F_{b-3} F_{x-2} F_{y-2} F_{c-1}+F_{l-2} F_{b-3} F_{x-2} F_{y-3} F_{c-2}+F_{l-2} F_{b-3} F_{y-2} F_{x-2} F_{c-1} \\
& +F_{l-2} F_{b-3} F_{y-2} F_{x-3} F_{c-2}+F_{l-2} F_{b-4} F_{x-2} F_{y-2} F_{c-2}+F_{l-3} F_{b-2} F_{x-2} F_{y-2} F_{c-1}
\end{aligned}
$$




$$
\begin{aligned}
& +F_{l-3} F_{b-2} F_{x-2} F_{y-3} F_{c-2}+F_{l-3} F_{b-3} F_{x-2} F_{y-2} F_{c-2}+F_{l-3} F_{b-2} F_{y-2} F_{x-2} F_{c-1} \\
& +F_{l-3} F_{b-2} F_{y-2} F_{x-3} F_{c-2}+F_{l-3} F_{b-3} F_{x-2} F_{y-2} F_{c-2}+F_{l-4} F_{b-2} F_{x-2} F_{y-2} F_{c-2} \\
& -\left(F_{x+y-3} F_{l-2} F_{b-3} F_{c-2}+F_{x+y-3} F_{l-3} F_{b-2} F_{c-2}\right) \\
& =F_{l-2} F_{b-3} F_{x-2} F_{y-2} F_{c-1}+F_{l-2} F_{b-3} F_{x-2} F_{y-3} F_{c-2}+F_{l-2} F_{b-3} F_{y-2} F_{x-2} F_{c-1} \\
& +F_{l-2} F_{b-3} F_{y-2} F_{x-3} F_{c-2}+F_{l-2} F_{b-4} F_{x-2} F_{y-2} F_{c-2}+F_{l-3} F_{b-2} F_{x-2} F_{y-2} F_{c-1} \\
& +F_{l-3} F_{b-2} F_{x-2} F_{y-3} F_{c-2}+F_{l-3} F_{b-3} F_{x-2} F_{y-2} F_{c-2}+F_{l-3} F_{b-2} F_{y-2} F_{x-2} F_{c-1} \\
& +F_{l-3} F_{b-2} F_{y-2} F_{x-3} F_{c-2}+F_{l-3} F_{b-3} F_{x-2} F_{y-2} F_{c-2}+F_{l-4} F_{b-2} F_{x-2} F_{y-2} F_{c-2} \\
& -\left(F_{x-1} F_{y-2} F_{l-2} F_{b-3} F_{c-2}+F_{x-2} F_{y-3} F_{l-2} F_{b-3} F_{c-2}+F_{x-2} F_{y-1} F_{l-3} F_{b-2} F_{c-2}\right. \\
& \left.+F_{x-3} F_{y-2} F_{l-3} F_{b-2} F_{c-2}\right) \\
& =F_{l-2} F_{b-3} F_{x-2} F_{y-2} F_{c-1}+F_{l-2} F_{b-3} F_{y-2} F_{x-2} F_{c-1}+F_{l-2} F_{b-3} F_{y-2} F_{x-3} F_{c-2} \\
& +F_{l-2} F_{b-4} F_{x-2} F_{y-2} F_{c-2}+F_{l-3} F_{b-2} F_{x-2} F_{y-2} F_{c-1}+F_{l-3} F_{b-2} F_{x-2} F_{y-3} F_{c-2} \\
& +F_{l-3} F_{b-3} F_{x-2} F_{y-2} F_{c-2}+F_{l-3} F_{b-2} F_{y-2} F_{x-2} F_{c-1}+F_{l-3} F_{b-3} F_{x-2} F_{y-2} F_{c-2} \\
& +F_{l-4} F_{b-2} F_{x-2} F_{y-2} F_{c-2}-\left(F_{x-1} F_{y-2} F_{l-2} F_{b-3} F_{c-2}+F_{x-2} F_{y-1} F_{l-3} F_{b-2} F_{c-2}\right) \\
& =F_{l-2} F_{b-3} F_{x-2} F_{y-2} F_{c-1}+F_{l-2} F_{b-3} F_{y-2} F_{x-2} F_{c-1}+F_{l-2} F_{b-3} F_{y-2} F_{x-3} F_{c-2} \\
& +F_{l-2} F_{b-4} F_{x-2} F_{y-2} F_{c-2}+F_{l-3} F_{b-2} F_{x-2} F_{y-2} F_{c-1}+F_{l-3} F_{b-2} F_{x-2} F_{y-3} F_{c-2} \\
& +F_{l-3} F_{b-3} F_{x-2} F_{y-2} F_{c-2}+F_{l-3} F_{b-2} F_{y-2} F_{x-2} F_{c-1}+F_{l-3} F_{b-3} F_{x-2} F_{y-2} F_{c-2} \\
& +F_{l-4} F_{b-2} F_{x-2} F_{y-2} F_{c-2}-\left(F_{x-2} F_{y-2} F_{l-2} F_{b-3} F_{c-2}+F_{x-3} F_{y-2} F_{l-2} F_{b-3} F_{c-2}\right. \\
& \left.+F_{x-2} F_{y-2} F_{l-3} F_{b-2} F_{c-2}+F_{x-2} F_{y-3} F_{l-3} F_{b-2} F_{c-2}\right) \\
& =F_{l-2} F_{b-3} F_{x-2} F_{y-2} F_{c-1}+F_{l-2} F_{b-3} F_{y-2} F_{x-2} F_{c-1}+F_{l-2} F_{b-4} F_{x-2} F_{y-2} F_{c-2} \\
& +F_{l-3} F_{b-2} F_{x-2} F_{y-2} F_{c-1}+F_{l-3} F_{b-3} F_{x-2} F_{y-2} F_{c-2}+F_{l-3} F_{b-2} F_{y-2} F_{x-2} F_{c-1} \\
& +F_{l-3} F_{b-3} F_{x-2} F_{y-2} F_{c-2}+F_{l-4} F_{b-2} F_{x-2} F_{y-2} F_{c-2}-\left(F_{x-2} F_{y-2} F_{l-2} F_{b-3} F_{c-2}\right. \\
& \left.+F_{x-2} F_{y-2} F_{l-3} F_{b-2} F_{c-2}\right) \\
& =\left(F_{l-2} F_{b-3} F_{x-2} F_{y-2} F_{c-1}-F_{x-2} F_{y-2} F_{l-2} F_{b-3} F_{c-2}\right)+F_{l-2} F_{b-3} F_{y-2} F_{x-2} F_{c-1} \\
& +F_{l-2} F_{b-4} F_{x-2} F_{y-2} F_{c-2}+\left(F_{l-3} F_{b-2} F_{x-2} F_{y-2} F_{c-1}-F_{x-2} F_{y-2} F_{l-3} F_{b-2} F_{c-2}\right) \\
& +F_{l-3} F_{b-3} F_{x-2} F_{y-2} F_{c-2}+F_{l-3} F_{b-2} F_{y-2} F_{x-2} F_{c-1}+F_{l-3} F_{b-3} F_{x-2} F_{y-2} F_{c-2} \\
& +F_{l-4} F_{b-2} F_{x-2} F_{y-2} F_{c-2} \\
& =F_{l-2} F_{b-3} F_{x-2} F_{y-2} F_{c-3}+F_{l-2} F_{b-3} F_{y-2} F_{x-2} F_{c-1}+F_{l-2} F_{b-4} F_{x-2} F_{y-2} F_{c-2} \\
& +F_{l-3} F_{b-2} F_{x-2} F_{y-2} F_{c-3}+F_{l-3} F_{b-3} F_{x-2} F_{y-2} F_{c-2}+F_{l-3} F_{b-2} F_{y-2} F_{x-2} F_{c-1} \\
& +F_{l-3} F_{b-3} F_{x-2} F_{y-2} F_{c-2}+F_{l-4} F_{b-2} F_{x-2} F_{y-2} F_{c-2} \\
& \geqslant F_{l-2} F_{b-3} F_{y-2} F_{x-2} F_{c-1}>0 \text {. }
\end{aligned}
$$

The last inequality follows by $m>3, b, c, x, y>2$ and $b c>6$. Hence, we get $z\left(\bar{H}_{m, b, c}^{k, x, y}\right)>$ $z\left(H_{m, l, b, c}^{k}\right)$. In view of $(2.5)$, we have $z(G) \geqslant z\left(H_{m, l, b, c}^{k}\right)$, the equality holds if and only if $G \cong H_{m, l, b, c}^{k}$.

By an argument similar to that in the proof of (a), we can also show that (b), (c) hold, respectively. This completes the proof of Lemma 2.9 .

Similar to Lemma 2.8, we have 
Lemma 2.10. For positive integers $m, l, b, c, k$,

(i) $z\left(H_{m, l-1, b, c}^{k+1}\right)<z\left(H_{m, l, b, c}^{k}\right)$ for either $l \geqslant 4, m \geqslant 3, b, c \geqslant 2$ and $b c \geqslant 6$, or $l=$ $3, m, b, c \geqslant 3$.

(ii) $z\left(H_{m-1, l, b, c}^{k+1}\right)<z\left(H_{m, l, b, c}^{k}\right)$ for $m \geqslant 4, l, b, c \geqslant 2$ and $l b c \geqslant 18$.

(iii) $z\left(H_{m, l, b-1, c}^{k+1}\right)<z\left(H_{m, l, b, c}^{k}\right)$ for either $b \geqslant 4, m \geqslant 3, l, c \geqslant 2$ and $l c \geqslant 6$, or $b=$ $3, m, l, c \geqslant 3$.

(iv) $z\left(H_{m, l, b, c-1}^{k+1}\right)<z\left(H_{m, l, b, c}^{k}\right)$ for either $c \geqslant 4, m \geqslant 3, b, c \geqslant 2$ and $l b \geqslant 6$, or $c=3, l, b, c \geqslant$ 3 .
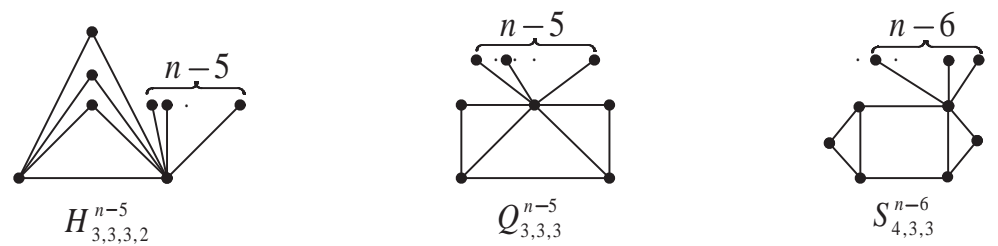

Figure 5: Graphs $H_{3,3,3,2}^{n-5}, Q_{3,3,3}^{n-5}$ and $S_{4,3,3}^{n-6}$

The following corollary follows by repeated applications of Lemma 2.10.

Corollary 2.11. Let $G \in \mathscr{T}_{n}^{6}$.

(i) If the arrangement of its six cycles is the same as Figure $4(I)$, then $z(G) \geqslant z\left(H_{3,3,3,2}^{n-5}\right)$, the equality holds if and only if $G \cong H_{3,3,3,2}^{n-5}$; see Figure 5.

(ii) If the arrangement of its six cycles is the same as Figure $4(I I)$, then $z(G) \geqslant z\left(Q_{3,3,3}^{n-5}\right)$, the equality holds if and only if $G \cong Q_{3,3,3}^{n-5}$; see Figure 5 .

(iii) If the arrangement of its six cycles is the same as Figure $4(I I I)$, then $z(G) \geqslant z\left(S_{4,3,3}^{n-6}\right)$, the equality holds if and only if $G \cong S_{4,3,3}^{n-6}$; see Figure 5 .

If $G \in \mathscr{T}_{n}^{7}$, then the arrangement of its seven cycles is depicted as Figure 6(i); see $[9,10,17,18,19]$. Let $R_{l, b, c, d}^{k, t_{1}, t_{2}}$ be a tricyclic graph on $n$ vertices (as shown in Figure 6(ii)), where $l+b+c+d+k+t_{1}+t_{2}=n+8$. Using a similar method as in Lemmas 2.9-2.10,

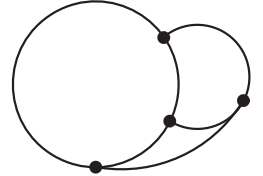

(i)

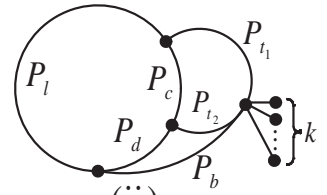

(ii)

Figure 6: The arrangement of the seven cycles in $\mathscr{T}_{n}^{7}$

we can obtain the following results, we omit the procedure here.

Lemma 2.12. Let $G \in \mathscr{T}_{n}^{7}$ such that the arrangement of its seven cycles is the same as Figure 6(ii), then we have $z(G) \geqslant z\left(R_{l, b, c, d}^{k, t_{1}, t_{2}}\right)$, where graph $R_{l, b, c, d}^{k, t_{1}, t_{2}}$ is from Figure 6(ii). 
Lemma 2.13. Given positive integers $l, t_{1}, t_{2}, b, c, d, k$, we have

(i) $z\left(R_{l-1, b, c, d}^{k+1, t_{1}, t_{2}}\right)<z\left(R_{l, b, c, d}^{k, t_{1}, t_{2}}\right)$ for $l \geqslant 3, t_{1}, t_{2}, b, c, d \geqslant 2$.

(ii) $z\left(R_{l, b-1, c, d}^{k+1, t_{1}, t_{2}}\right)<z\left(R_{l, b, c, d}^{k, t_{1}, t_{2}}\right)$ for $b \geqslant 3, l, t_{1}, t_{2}, b, c, d \geqslant 2$.

(iii) $z\left(R_{l, b, c-1, d}^{k+1, t_{1}, t_{2}}\right)<z\left(R_{l, b, c, d}^{k, t_{1}, t_{2}}\right)$ for $c \geqslant 3, l, t_{1}, t_{2}, b, d \geqslant 2$.

(iv) $z\left(R_{l, b, c, d-1}^{k+1, t_{1}, t_{2}}\right)<z\left(R_{l, b, c, d}^{k, t_{1}, t_{2}}\right)$ for $d \geqslant 3, l, t_{1}, t_{2}, c \geqslant 2$.

(v) $z\left(R_{l, b, c, d}^{k+1, t_{1}-1, t_{2}}\right)<z\left(R_{l, b, c, d}^{k, t_{1}, t_{2}}\right)$ for $t_{1} \geqslant 3, l, t_{2}, b, c, d \geqslant 2$.

(vi) $z\left(R_{l, b, c, d}^{k+1, t_{1}, t_{2}-1}\right)<z\left(R_{l, b, c, d}^{k, t_{1}, t_{2}}\right)$ for $t_{2} \geqslant 3, l, t_{1}, b, c, d \geqslant 2$.

\section{Main results}

In this section, we determine a sharp lower bound for the Hosoya index of tricyclic graphs in $\mathscr{T}_{n}$, the corresponding extremal graph is characterized.
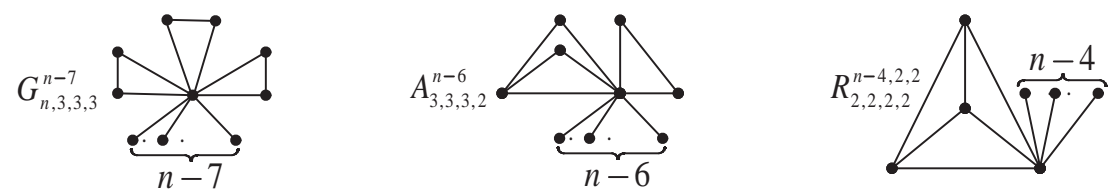

Figure 7: Graphs $G_{n, 3,3,3}^{n-7}, A_{3,3,3,2}^{n-6}$ and $R_{2,2,2,2}^{n-4,2,2}$

Proposition 3.1. Let $G \in \mathscr{T}_{n}^{3}$, then $z(G) \geqslant z\left(G_{n, 3,3,3}^{n-7}\right)$, the equality holds if and only if $G \cong G_{n, 3,3,3}^{n-7} ;$ see Figure 7 .

Proof. It is a direct consequence of Lemmas 2.5 and 2.6.

Repeated applications of Lemma 2.8 give the following proposition.

Proposition 3.2. Let $G \in \mathscr{T}_{n}^{4}$, then $z(G) \geqslant z\left(A_{3,3,3,2}^{n-6}\right)$, and the equality holds if and only if $G \cong A_{3,3,3,2}^{n-6} ;$ see Figure $\%$.

Proposition 3.3. Let $G \in \mathscr{T}_{n}^{6}$, then $z(G) \geqslant z\left(H_{3,3,3,2}^{n-5}\right)$, and the equality holds if and only if $G \cong H_{3,3,3,2}^{n-5}$; see Figure 5 .

Proof. By Corollary 2.11, we have $z(G) \geqslant \min \left\{z\left(H_{3,3,3,2}^{n-5}\right), z\left(Q_{3,3,3}^{n-5}\right), z\left(S_{4,3,3}^{n-6}\right)\right\}$. By direct calculation, we get $z\left(H_{3,3,3,2}^{n-5}\right)=4 n-6, z\left(Q_{3,3,3}^{n-5}\right)=5 n-10, z\left(S_{4,3,3}^{n-6}\right)=10 n-35$, hence we obtain the desired results.

Repeated applications of Lemma 2.13 give the following proposition.

Proposition 3.4. Let $G \in \mathcal{T}_{n}^{7}$, then $z(G) \geqslant z\left(R_{2,2,2,2}^{n-4,2,2}\right)$, the equality holds if and only if $G \cong R_{2,2,2,2}^{n-4,2,2} ;$ see Figure $\%$.

Summarizing Propositions 3.1, 3.2, 3.3 and 3.4, we arrive at: 
Theorem 3.5. Let $G \in \mathscr{T}_{n}$, then $z(G) \geqslant 4 n-6$, the equality holds if and only if $G \cong$ $H_{3,3,3,2}^{n-5}$ (see Figure 5), or $R_{2,2,2,2}^{n-4,2,2}$ (see Figure 7).

Proof. By Propositions 3.1, 3.2, 3.3 and 3.4, for any $G \in \mathscr{T}_{n}$,

$$
z(G) \geqslant \min \left\{z\left(G_{n, 3,3,3}^{n-7}\right), \quad z\left(A_{3,3,3,2}^{n-6}\right), \quad z\left(H_{3,3,3,2}^{n-5}\right), \quad z\left(R_{2,2,2,2}^{n-4,2,2}\right)\right\} .
$$

Note that

$z\left(G_{n, 3,3,3}^{n-7}\right)=8 n-24, \quad z\left(A_{3,3,3,2}^{n-6}\right)=6 n-14, \quad z\left(H_{3,3,3,2}^{n-5}\right)=4 n-6, \quad z\left(R_{2,2,2,2}^{n-4,2,2}\right)=4 n-6$,

hence, $z(G) \geqslant 4 n-6$. By Propositions 3.3 and 3.4 the equality holds if and only if $G \cong H_{3,3,3,2}^{n-5}$ or, $R_{2,2,2,2}^{n-4,2,2}$.

\section{Conclusion remark}

In this paper, we have determined the sharp lower bound on the total number of matchings of tricyclic graphs on $n$ vertices. It is surprised to see that the graph of $n$-vertex tree (unicyclic graph, bicyclic graph) which attains the smallest Hosoya index is unique, while our result on $n$-vertex tricyclic graphs, the extremal graph which attains the smallest Hosoya index is not unique. On the other hand, it is natural to consider the following problem which may be much more difficult.

Problem 4.1. How can we determine a sharp upper bound on the total number of matchings of tricyclic graphs with $n$ vertices?

Acknowledgments. The authors would like to express their sincere gratitude to the referee for a very careful reading of the paper and for all his or her insightful comments and valuable suggestions, which led to a number of improvements in this paper.

\section{References}

[1] B. Bollobás, Modern Graph Theory (Springer-Verlag, 1998).

[2] O. Chan, I. Gutman, T.K. Lam, R. Merris, Algebraic connections between topological indices, J. Chem. Inform. Comput. Sci. 38(1998) 62-65.

[3] Y.Q. Chen, L.G. Wang, The Laplacian spread of tricyclic graphs. Electron. J. Combin. 16 (1) (2009), Research Paper 80, 18 pp.

[4] S.J. Cyvin, I. Gutman, Hosoya index of fused molecules, MATCH Commun. Math. Comput. Chem. 23 (1988) 89-94.

[5] S.J. Cyvin, I. Gutman, N. Kolakovic, Hosoya index of some polymers, MATCH Commun. Math. Comput. Chem. 24(1989) 105-117.

[6] H. Deng, S. Chen, The extremal unicyclic graphs with respect to Hosoya index and Merrifield-Simmons index, MATCH Commun. Math.Comput. Chem. 59 (2008) 171-190.

[7] H. Deng, The smallest Hosoya index in $(n, n+1)$-graphs, J. Math. Chem. 43 (1) (2008) 119-133. 
[8] H. Deng, The largest Hosoya index of $(n, n+1)$-graphs, Comput. Math. Appl. 56 (10) (2008) 2499-2506.

[9] X. Geng, S. Li, X. Li, On the index of tricyclic graphs with perfect matchings, Linear Algebra Appl. 431 (2009) 2304-2316.

[10] X. Geng, S. Li, The spectral radius of tricyclic graphs with $n$ vertices and $k$ pendant vertices, Linear Algebra Appl. 428 (11-12) (2008) 2639-2653.

[11] S.G. Guo, Y.F. Wang, The Laplacian spectral radius of tricyclic graphs with $n$ vertices and $k$ pendant vertices, Linear Algebra Appl. 431 (1-2) (2009) 139-147.

[12] I. Gutman, O.E. Polansky, Mathematical Concepts in Organic Chemistry, Springer, Berlin, 1986.

[13] H. Hosoya, Topological index, a newly proposed quantity characterizing the topological nature of structural isomers of saturated hydrocarbons, Bull. Chem. Soc. Jpn. 44 (1971) 2332-2339.

[14] Y. Hou, On acyclic systems with minimal Hosoya index, Discrete Appl. Math. 119 (2002) 251-257.

[15] H. Hua, Minimizing a class of unicyclic graphs by means of Hosoya index, Math. Comput. Modelling. 48 (2008) 940-948.

[16] H. Hua, Hosoya index of unicyclic graphs with prescribed pendant vertices, J. Math. Chem. 43 (2008) 831-844.

[17] S. Li, On the nullity of graphs with pendant vertices, Linear Algebra Appl. 429 (7) (2008) 1619-1628.

[18] S. Li, X. Li, On tricyclic graphs of a given diameter with minimal energy, Linear Algebra Appl. 430 (2009) 370-385.

[19] S. Li, X. Li, and Z. Zhu, On tricyclic graphs with minimal energy, MATCH Commun. Math. Comput. Chem. 59 (2008) 397-419.

[20] S. Li, X. Li, W. Jing, On the extremal Merrifield-Simmons index and Hosoya index of quasi-tree graphs, Discrete Appl. Math. 157 (2009) 2877-2885.

[21] S. Li, X. Li, Z. Zhu, On minimal energy and Hosoya index of unicyclic graphs, MATCH Commun. Math. Comput. Chem. 61 (2009) 325-339.

[22] H. Liu, The proof of a conjecture concerning acyclic molecular graphs with maximal Hosoya index and diameter 4, J. Math. Chem. 43 (2008) 1199-1206.

[23] H. Liu and M. Lu, A unified approach to extremal cacti for different indices, MATCH Commun. Math. Comput. Chem. 58 (2007) 193-204.

[24] H. Liu, X. Yan and Z. Yan, On the Merrifield-Simmons indices and Hosoya indices of trees with a prescribed diameter, MATCH Commun. Math. Comput. Chem. 57 (2007) 371-384.

[25] Z. Machnicka, A. Włoch, I. Włoch, Bounds of the Hosoya index in graphs, AKCE Int. J. Graphs Comb. 5 (2008) 181-187.

[26] R.E. Merrifield and H.E. Simmons, Topological Methods in Chemistry (Wiley, New York, 1989).

[27] J. Ou, On extremal unicyclic molecular graphs with maximal Hosoya index, Discrete Appl. Math. 157 (2009) 391-397.

[28] J. Ou, Maximal Hosoya index and extremal acyclic molecular graphs without perfect matching, Appl. Math. Lett. 19 (2006) 652-656.

[29] J. Ou, On acyclic molecular graphs with maximal Hosoya index, energy, and short diameter, J. Math. Chem. 43 (2008) 328-337.

[30] H. Ren, F. Zhang, Double hexagonal chains with maximal Hosoya index and minimal Merrifield-Simmons index, J. Math. Chem. 42 (2007) 679-690. 
[31] W.C. Shiu, Extremal Hosoya index and Merrifield-Simmons index of hexagonal spiders, Discrete Appl. Math. 156 (2008) 2978-2985.

[32] L. Türker, Contemplation on the Hosoya indices, J. Mol. Struc. (THEOCHEM), 623 (2003) 75-77.

[33] A. Yu, X. Lv, The Merrifield-Simmons indices and Hosoya indices of trees with $k$ pendant vertices, J. Math. Chem. 41 (2007) 33-43. 